\title{
Survivable IP/MPLS-Over-WSON Multilayer Network Optimization
}

\author{
M. Ruiz, O. Pedrola, L. Velasco, D. Careglio, J. Fernández-Palacios, and G. Junyent
}

\begin{abstract}
Network operators are facing the problem of dimensioning their networks for the expected huge IP traffic volumes while keeping constant or even reducing the connectivity prices. Therefore, new architectural solutions able to cope with the expected traffic increase in a more cost-effective way are needed. In this work, we study the survivable IP/multi-protocol label switching (MPLS) over wavelength switched optical network (WSON) multilayer network problem as a capital expenditure (CAPEX) minimization problem. Two network approaches providing survivability against optical links, IP/MPLS nodes, and opto-electronic port failures are compared: the classical overlay approach where two redundant IP/MPLS networks are deployed, and the new joint multilayer approach which provides the requested survivability through an orchestrated interlayer recovery scheme which minimizes the over-dimensioning of IP/MPLS nodes. Mathematical programming models are developed for both approaches. Solving these models, however, becomes impractical for realistic networks. In view of this, evolutionary heuristics based on the biased random-key genetic algorithm framework are also proposed. Exhaustive experiments on several reference network scenarios illustrate the effectiveness of the proposed approach in minimizing network CAPEX.
\end{abstract}

Index Terms-Integer linear programming; Multilayer planning; Survivable multilayer networks.

\section{INTRODUCTION}

$\mathbf{I}$ $\mathrm{n}$ recent years, core transport networks have evolved from synchronous optical networking (SONET)/synchronous digital hierarchy (SDH) over static point-to-point dense wavelength division multiplexing (DWDM) links toward wavelength switched optical networks (WSONs) introducing dynamic reconfiguration, i.e., automatic optical connection (lightpath) setup and tear-down and recovery mechanisms such as protection and restoration. WSON transparency and dynamicity reduce both costs and power consumption in core transport networks. The benefits achieved are significantly increased by applying an intelligent interworking strategy between IP/multi-protocol label switching (MPLS) networks and WSONs based on a multilayer optimization process capable of efficiently aggregating the various bandwidth granularities. In this work, we tackle the issue of planning an IP/MPLS network specifically tailored to provide layer 1

Manuscript received February 14, 2011; revised May 18, 2011; accepted July 4, 2011; published July 29, 2011 (Doc. ID 142594).

M. Ruiz (e-mail: mruiz@ac.upc.edu), O. Pedrola, L. Velasco, D. Careglio, and G. Junyent are with the Advanced Broadband Communications Center (CCABA), Universitat Politècnica de Catalunya (UPC), Barcelona, Spain.

J. Fernández-Palacios is with Telefónica I+D, Madrid, Spain.

Digital Object Identifier 10.1364/JOCN.3.000629
(L1) and L2 virtual private network services for companies, i.e., those services that can be classified as premium services and thus requiring the highest availability.

Traditionally, only optical link and opto-electronic (OE) ports have been considered as points of failure and, consequently, networks include protection or restoration mechanisms to survive these failures. Nonetheless, IP/MPLS nodes are not always as reliable as traditional telecom equipment due to the constant software and hardware upgrades [1,2]. Therefore, operators usually protect their IP/MPLS networks against node failures by duplicating backbone nodes (i.e., applying redundancy) and thus highly increasing network capital expenditure (CAPEX), i.e., those costs associated with purchasing and installing fixed infrastructures, such as equipment [1]. Notwithstanding, other recovery schemes different from redundancy can be designed for multilayer networks, e.g., the authors in [2] present a tutorial of multilayer recovery schemes. The authors in [3] propose and evaluate a coordinated link restoration scheme to be used in packet-over-optical networks. Although the proposed scheme is cost effective compared with duplicating nodes, it requires the IP/MPLS and the optical topologies to be symmetrical, i.e., every node has both packet and optical switching capacities. However, the underlying WSON, constituting the core of the transport network, has to support heterogeneous client networks and a wide range of services to residential and business customers, needing thus to provide different availability degrees.

In this paper we face the Survivable IP/MPLS-over-WSON MULTilAyer NEtwork Optimization (hereafter SIMULTANEO) problem. To this end, two approaches are compared: our joint approach consisting in over-dimensioning backbone IP/MPLS nodes and applying lightpath and connectivity restoration, and the overlay approach consisting in duplicating backbone IP/MPLS nodes. For the joint approach, orchestrated interlayer recovery actions are defined to avoid IP/MPLS backbone node duplication. Since no symmetrical topologies are needed, the WSON is allowed to serve several client networks with different availability needs. Moreover, we rely on lightpath restoration which provides a finer granularity to recover selected lightpaths in really short times (e.g., hundreds of milliseconds [4]). Besides lightpath restoration, a novel connectivity restoration scheme is also proposed to deal, not only with IP/MPLS node failures, but also with all other types of failure.

Some other works have studied the IP/MPLS-over-WSON multilayer network planning problem and present different integer linear programming (ILP) models and/or heuristic algorithms (e.g., [5-11]). The authors in [5] investigate traffic 
grooming in mesh networks with the objective of improving the network throughput. On the contrary, our objective is to minimize network CAPEX for a given demand matrix. The authors in [6] propose hierarchical traffic grooming, defining clusters of nodes and selecting one of them as a hub. Hub nodes are responsible for grooming traffic before leaving the cluster. In [7], the authors develop an algorithm to find optimal clusters. Although our approach also takes advantage of hierarchical grooming, we do not limit nodes to having only one hub, creating a virtual star; instead we allow a node to be connected to several hubs. The authors in [8] present an ILP formulation to maximize a utility function for the network operator (i.e., the difference between revenues and costs), but failures are not considered. To solve the problem, a Lagrangian relaxationbased method is proposed. A similar approach does not work for the SIMULTANEO problem as a consequence of its size; it includes a huge set of single-failure scenarios (i.e., every IP/MPLS node, OE port, and optical link in the network). In view of this, a biased random-key genetic algorithm (BRKGA)based heuristic [9] is here proposed to solve SIMULTANEO in practical running times. Previous works have proposed evolutionary genetic algorithms (GAs) for optical network planning. The authors in [10] present a GA-based heuristic for single layer survivable optical network planning, whereas the authors in [11] apply GAs to dimension single layer dynamic optical networks. In contrast, we use the novel BRKGA meta-heuristic to solve the overall SIMULTANEO problem.

Finally, note that the coordinated recovery actions that we propose in this paper could be implemented using a common control plane based on the generalized multi-protocol label switching (GMPLS) protocol suite [12]. As an example, the authors in [13] propose a multilayer routing approach to be used in symmetric GMPLS-controlled multilayer networks and compare it with an approach where each layer applies its own routing policy.

The remainder of this paper is organized as follows. Section II describes the SIMULTANEO problem and presents two different network approaches: joint and overlay. An ILP model for the joint multilayer network planning is presented in Section III. For comparison purposes, an ILP-based method for the overlay network planning is also provided. Due to the fact that the ILP models are computationally impractical when realistic problem instances are considered, heuristic algorithms able to obtain near-optimal solutions to the problem are developed in Section IV. Using these heuristics, the performances of both the joint and the overlay network approaches are compared in Section V in terms of CAPEX. Finally, Section VI concludes the paper.

\section{Survivable Multilayer Network Design}

Three types of nodes can be distinguished at the IP/MPLS packet layer: metro nodes performing client flow aggregation, transit nodes providing routing flexibility, and interconnection nodes supporting inter-operator connections. To minimize the number of ports, metro-to-metro connections are avoided being as every metro node is connected to one or more optical cross-connects (OXCs). Moreover, while it is typical that a transit node is collocated with an OXC, metro nodes are usually
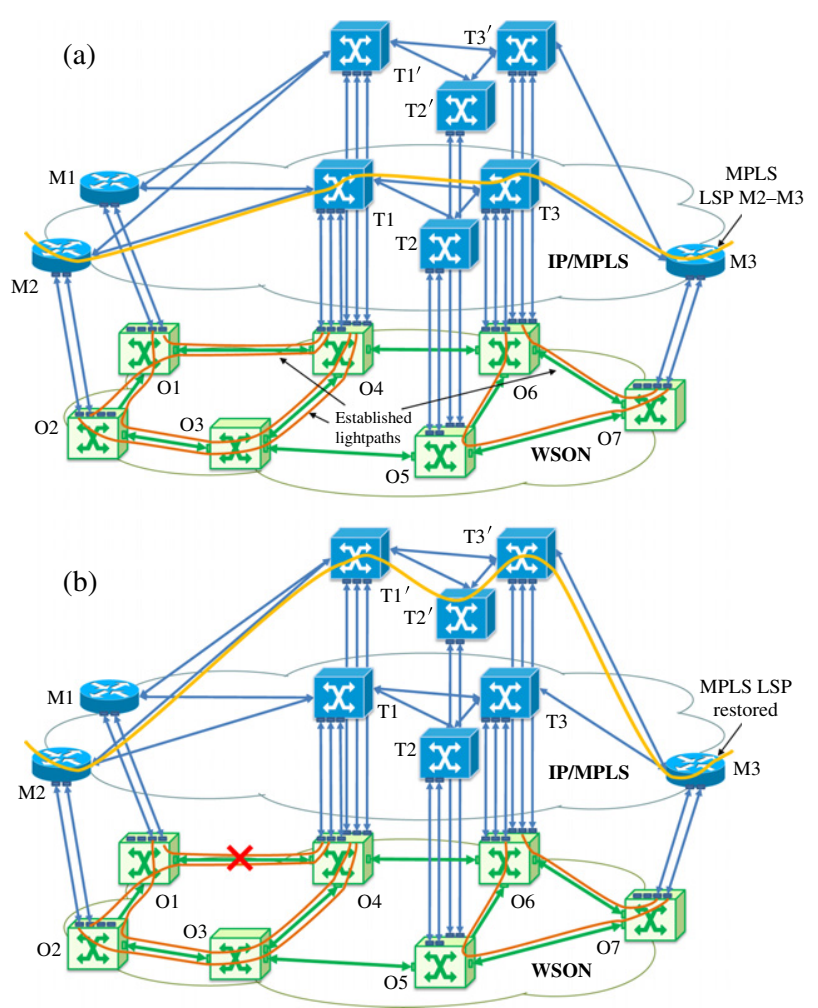

Fig. 1. (Color online) (a) The overlay network approach, (b) single link/node failure recovery.

closer to clients, and, thus, it is likely that some ad hoc connectivity needs to be used to connect metro nodes to OXCs. The IP/MPLS network must provide transport service to all demands, even in the event of any single failure. Specifically, we consider optical link cuts and IP/MPLS node and OE port failures.

From the IP/MPLS network perspective, network planning could be faced keeping layer independence following an overlay approach. In this case, recovery mechanisms are kept within each layer and hence no interlayer recovery mechanisms are required. For this reason, IP/MPLS node and virtual link redundancy must be foreseen at the IP/MPLS layer to prevent outages. As an example, Fig. 1(a) illustrates a design for an IP/MPLS network with redundancy against failures, where transit nodes are duplicated creating two parallel topologies. Note that in Fig. 1(a) transit node $\mathrm{T} 1^{\prime}$ is the redundant node of T1. If an optical link fails (O1-O4 in Fig. 1(b)), the affected MPLS label switched path (LSP) between metro nodes M2 and M3 is restored using connectivity capacity in the redundant topology. Obviously this scheme, although providing optimal network dimensioning for both layer networks separately, is far from achieving an optimal overall cost reduction.

On the contrary, specifically designed restoration mechanisms which are able to trigger coordinated actions across the two layers can be applied to avoid IP/MPLS node duplication; for example, those defined in [3] for symmetrical multilayer networks. Figure 2 depicts an example of our joint approach designed for non-symmetrical environments that illustrates how node and link redundancy can be avoided. Figure 2(a) shows a multilayer network where each IP/MPLS metro 
(a)

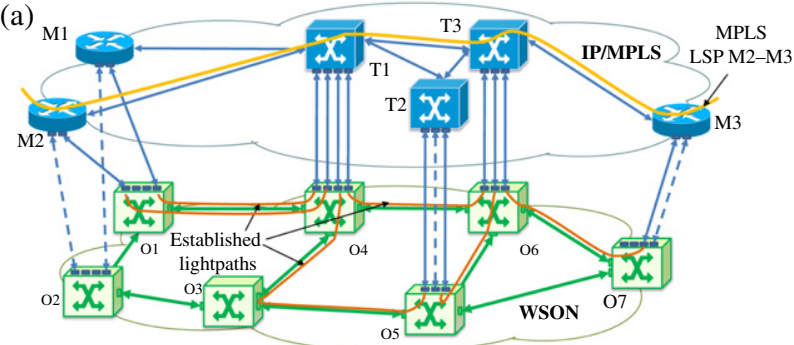

(b)
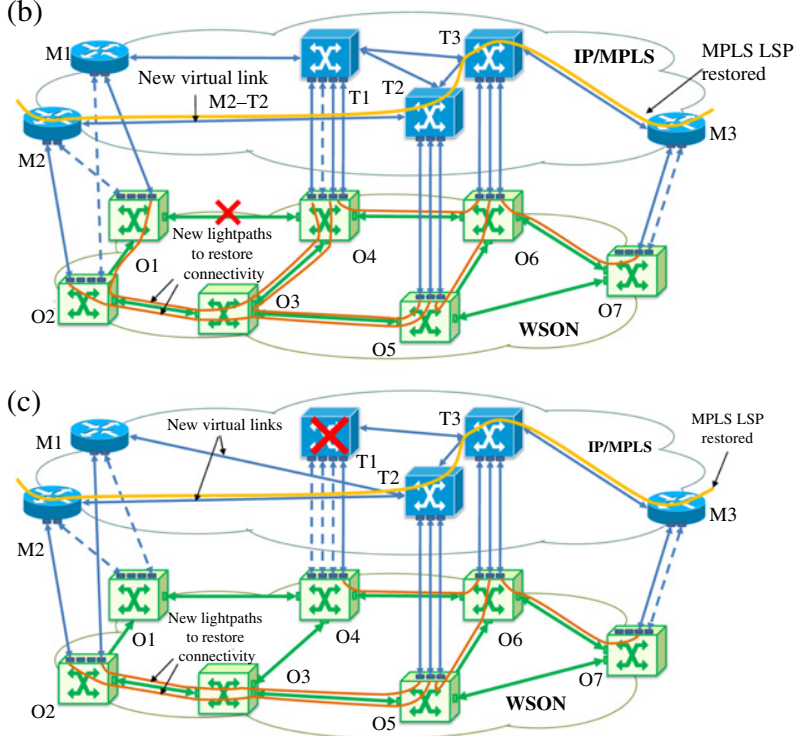

Fig. 2. (Color online) (a) Joint approach, (b) link failure recovery, and (c) node failure recovery.

node is connected to a transit node through virtual links creating a virtual topology. Each virtual link is supported by a lightpath routed through the minimum path cost over the WSON network. In the example, metro nodes M1 and M2 are connected to transit node T1 and metro node M3 to transit node T3 through only one lightpath. Note that in order to guarantee failure recovery, extra capacity has been added in every node (dotted lines in Fig. 2).

When an optical link fails, the multilayer network applies joint recovery schemes to restore the affected traffic. For instance, when optical link O1-O4 fails (Fig. 2(b)), recovery actions are taken to restore metro-to-transit connectivity. If a lightpath can be restored within the optical layer, the connectivity at the IP/MPLS remains unaltered (virtual link $\mathrm{M} 1-\mathrm{T} 1$ is restored through lightpath O1-O2-O3-O4). On the contrary, a new lightpath needs to be established to connect the IP/MPLS metro node to a different transit node, thus restoring the metro-to-transit connectivity (virtual link M2-T2 through new lightpath O2-O3-O5). Once the connectivity is restored, MPLS LSPs can be eventually rerouted over the reconfigured virtual topology.

In the event of an OE port failure, spare OE ports are added. Then, the affected lightpath can be torn down and set up again on a different OE port. Finally, when an IP/MPLS node fails (Fig. 2(c)), new lightpaths are established between every metro node connected to the failed node and a different transit node, so as to restore the metro-to-transit connectivity, thus creating new virtual links. In the example, virtual links M1-T2 and M2-T2 are created. Once the connectivity is restored, the affected MPLS LSPs are rerouted.

Additionally, the introduction of WSON allows the performance of optical by-passing, i.e., highly loaded metro-tometro and metro-to-interconnection connectivity is performed directly at the optical layer without intermediate IP/MPLS routing. The gains are multi-fold: the number of $\mathrm{OE}$ interfaces as well as the switching capacity of the IP/MPLS nodes is kept to the absolute minimum, and, hence, power consumption is kept on low/moderate levels. Although optical by-passing in general can reduce network costs, its use has been restricted to just highly loaded virtual links to avoid MAC address table explosion [14].

The next section is devoted first to formally stating the SIMULTANEO problem and second to presenting ILP-based models for both the joint and the overlay approaches.

\section{MATHEMATICAL FORMULATION}

\section{A. SIMULTANEO Problem Statement}

The problem can be formally stated as follows:

Given:

- an optical network, represented by a graph $G_{O}\left(N, L_{O}\right)$, $N$ being the set of OXCs and $L_{O}$ the set of fiber links connecting two OXCs;

- a set $W L$ of available wavelengths in each link in $L_{O}$;

- the virtual network represented by a graph $G_{V}(V, E), V$ being the set of IP/MPLS nodes and $E$ the set of virtual links defining the connectivity among IP/MPLS nodes;

- a set $L_{R}$ containing the fiber links connecting an IP/MPLS node to an OXC;

- a set $D$ of IP/MPLS demands to be transported;

- equipment cost, specified by a fixed cost for every type of IP/MPLS node and OE port;

- cost per $\mathrm{km}$ of using the already deployed fiber, depending on the lightpath recovery type (e.g., unprotected, restorable, etc.).

\section{Output:}

- the configuration of every IP/MPLS node in terms of switching capacity and number and bit-rate of OE ports;

- the set of OE ports in every OXC;

- the set of used virtual links, including its route over the WSON;

- the route of every demand over the virtual topology.

Objective: Minimize the expected CAPEX for the network designed for the given set of demands.

As previously discussed, the problem can be faced using two different network planning approaches. For each approach, an ILP-based model is provided next. 


\section{B. ILP Model for the Joint Approach}

The model for the joint approach optimizes both the IP/MPLS and WSON layers simultaneously by adding extra capacity to overcome any single failure. It combines node-link and arc-path formulations respectively for IP/MPLS grooming, routing, and network planning, and WSON routing given a set of pre-computed optical routes. IP/MPLS demands are routed through a virtual topology, where each virtual link is divided into several channels. Each channel aggregates a number of IP/MPLS demands and is transported over a single lightpath in the WSON. For each channel carrying demands, four OE ports of the same bit-rate must be equipped (two at the end IP/MPLS nodes and two in the associated OXCs).

As mentioned above, in this work we consider IP/MPLS node, OE port, and fiber link failures. IP/MPLS nodes and fiber links are some of the invariants of the problem, i.e., they are known beforehand, and so the set of failures that may affect them is known. In contrast, the number and location of OE ports are some of the outputs of the problem, and thus the failures that may affect them are unknown before the optimization. Owing to this fact, port failures may require the use of non-linear constraints given the quadratic form of the resulting constraints. To avoid such non-linearity, we have attached a pre-defined set of slots to each IP/MPLS node where each slot could contain one OE port. In this way, failures are related to slots instead of OE ports, and hence known in advance. Note that a failure in a non-equipped slot has null effect over the optimal solution.

For each failure $i$, one single-failure scenario is defined by characterizing the set of resources (IP/MPLS nodes, slots, virtual links, and WSON links) that can be used to reroute the affected traffic when the failure occurs. Channel-to-slot and channel-to-lightpath assignments are determined for each failure scenario. Although these assignments strongly increase the size of the problem, they provide flexibility to overcome any failure. It is worth mentioning that network dimensioning ensures that every demand is successfully transported under any single-failure scenario.

The following sets and parameters have been defined:

\footnotetext{
Optical topology:

$N \quad$ Set of OXCs.

$L \quad$ Set of fiber links, index $l . L=L_{O} \cup L_{R}$.

$L_{O} \quad$ Subset of $L$ connecting two OXCs.

$L_{R} \quad$ Subset of $L$ connecting an IP/MPLS node to an OXC.

$w_{l} \quad$ Number of wavelengths of fiber link $l \in L_{O}$.

$l e n_{l} \quad$ Length of fiber link $l \in L$ in kilometers.

$K \quad$ Set of WSON routes, index $k$.

path $_{l}^{k}$ Equal to 1 if route $k$ contains fiber link $l, 0$ otherwise.

Virtual topology:

$V \quad$ Set of IP/MPLS nodes, index $v$.

$V_{M} \quad$ Subset of $V$ containing the metro nodes.

$V_{T} \quad$ Subset of $V$ containing the transit nodes.

$V_{I} \quad$ Subset of $V$ containing the interconnection nodes.

$V_{V} \quad$ Subset of $V$ containing the virtual nodes.
}

\begin{tabular}{|c|c|}
\hline$S(v)$ & Set of slots in node $v$, index $s$. \\
\hline$E$ & Set of virtual links, index $e$. \\
\hline$E_{1}$ & $\begin{array}{l}\text { Subset of } E \text { containing metro-to-transit and } \\
\text { transit-to-transit virtual links. }\end{array}$ \\
\hline$E_{2}$ & Subset of $E$ containing metro-to-metro virtual links \\
\hline$E_{3}$ & $\begin{array}{l}\text { Subset of } E \text { containing transit-to-interconnection } \\
\text { and interconnection-to-virtual virtual links. }\end{array}$ \\
\hline$E_{4}$ & $\begin{array}{l}\text { Subset of } E \text { containing metro-to-interconnection } \\
\text { virtual links. }\end{array}$ \\
\hline$E_{5}$ & $\begin{array}{l}\text { Subset of } E_{3} \text { containing interconnection-to-virtual } \\
\text { virtual links. }\end{array}$ \\
\hline$E_{x}(v)$ & $\begin{array}{l}\text { Subset of virtual links belonging to subset } E_{x} \\
\text { incident to node } v \text {, for } x=1 \ldots 5 \text {. }\end{array}$ \\
\hline$I(e)$ & Set of end nodes of virtual link $e$, index $v$. \\
\hline$C(e)$ & Set of channels of virtual link $e$, index $c$. \\
\hline$K(e)$ & $\begin{array}{l}\text { Subset of } K \text { containing the WSON routes } \\
\text { connecting both ends of virtual link } e \text {. }\end{array}$ \\
\hline
\end{tabular}

Demands:

$D \quad$ Set of IP/MPLS demands, index $d$.

$D_{1} \quad$ Subset of $D$ with low and moderate data-rate demands.

$D_{2} \quad$ Subset of $D$ with high data-rate demands, susceptible to optical by-passing.

$S D(d) \quad$ Set of source and destination nodes of demand $d$.

$b_{d} \quad$ Bandwidth of demand $d$ in Gbps.

$h_{d} \quad$ Equal to 1 if demand $d$ belongs to subset $D_{2}, 0$ otherwise.

out $\quad$ Equal to 1 if an end node of demand $d$ belongs to $V_{V}, 0$ otherwise.

Failures:

$F \quad$ Set of failure scenarios, index $f$. Scenario 0

represents the scenario without failure.

$w$ fail $^{f k}$ Equal to 1 if WSON route $k$ is available under failure scenario $f, 0$ otherwise.

$m$ fail $^{f v s}$ Equal to 1 if slot $s$ of node $v$ is available under failure scenario $f$.

Equipment, costs, and others:

$\begin{array}{ll}P T & \text { Set of port bit-rates, index } i . \\ p k_{i} & \text { Capacity of a port of bit-rate } i \text { in Gbps. } \\ o p c_{i} & \text { Cost of one port of bit-rate } i \text { in an OXC. } \\ m p c_{i} & \text { Cost of one port of bit-rate } i \text { in an IP/MPLS node. } \\ R T & \text { Set of node classes, index } j . \text { Each class defined by a } \\ & \text { switching capacity and a number of slots. } \\ r k_{j} & \text { Switching capacity of a node class } j \text { in Gbps. } \\ r p k_{j} & \text { Number of slots available in a node class } j . \\ r c_{j} & \text { Cost of one node of class } j . \\ C_{r e s t} & \text { Cost per kilometer of restorable lightpath. } \\ M & \text { A large positive constant. }\end{array}$

The decision variables are

$\omega_{d e c}^{f} \quad$ Binary. Equal to 1 when demand $d$ is routed through channel $c$ of virtual link $e$, under failure scenario $f$. 0 otherwise.

$\gamma_{d}^{f} \quad$ Binary. Equal to 1 if the route of demand $d$ under failure scenario $f$ must be the same as that in the non-failure scenario. 0 otherwise. 
$\phi_{e c}^{f k} \quad$ Binary. Equal to 1 if channel $c$ of virtual link $e$ is assigned to WSON route $k$, under failure scenario $f$. 0 otherwise.

$\psi_{e c}^{f v s} \quad$ Binary. Equal to 1 if channel $c$ of virtual link $e$ is assigned to slot $s$ of node $v$, under failure scenario $f$. 0 otherwise.

$\rho_{i}^{v s} \quad$ Binary. Equal to 1 if slot $s$ of node $v$ is equipped with a port of bit-rate $i$. 0 otherwise.

$\pi_{j}^{v} \quad$ Binary. Equal to 1 if node $v$ is equipped with a node of class $j .0$ otherwise.

${ }_{\tau}{ }^{\text {fus }} \quad$ Positive integer for the total amount of traffic (in Gbps) in slot $s$ of node $v$ under failure scenario $f$.

Then, network CAPEX can be computed as the sum of the following expressions:

$$
\begin{aligned}
\operatorname{COST}_{\text {Equipment }}^{\text {Joint }}= & \sum_{v \in V \backslash V_{V}}\left(\sum_{s \in S(v)} \sum_{i \in P T}\left(m p c_{i}+o p c_{i}\right) \cdot \rho_{i}^{v s}\right. \\
& \left.+\sum_{j \in R T} r c_{j} \cdot \pi_{j}^{v}\right),
\end{aligned}
$$

$\operatorname{COST}_{\text {Lightpaths }}^{\text {Joint }}=C_{\text {rest }} \cdot \sum_{e \in E} \sum_{c \in C(e)} \sum_{k \in K(e)} \varphi_{e c}^{0 k} \cdot \sum_{l \in L} l e n_{l} \cdot p_{a t h}^{k}$,

where Eq. (1) computes the cost of IP/MPLS nodes and OE ports and Eq. (2) computes the cost of the lightpaths.

Finally, the ILP for the joint approach is as follows:

\section{SIMULTANEO-Joint}

$$
\text { Minimize } \mathrm{CAPEX}^{\text {Joint }}=\mathrm{COST}_{\text {Equipment }}^{\text {Joint }}+\mathrm{COST}_{\text {Lightpaths }}^{\text {Joint }}
$$

subject to:

$$
\begin{gathered}
\sum_{e \in E_{1}(v)} \sum_{c \in C(e)} \omega_{d e c}^{f}+h_{d} \cdot \sum_{e \in E_{2}(v)} \sum_{c \in C(e)} \omega_{d e c}^{f}=1 \\
\forall d \in D, f \in F, v \in S D(d) \cap \overline{V_{V}}, \\
\sum_{e \in E_{1}(v) \cup E_{3}(v) c \in C(e)} \omega_{d e c}^{f}+h_{d} \cdot \sum_{e \in E_{4}(v)} \sum_{c \in C(e)} \omega_{d e c}^{f}=1 \\
\forall d \in D, f \in F, v \in S D(d) \cap V_{V}, \\
\sum_{e \in E_{1}(v)} \sum_{c \in C(e)} \omega_{d e c}^{f}+o u t_{d} \cdot \sum_{e \in E_{3}(v)} \sum_{c \in C(e)} \omega_{d e c}^{f} \leq 2 \\
\forall d \in D, f \in F, v \in \overline{S D(d)} \cap\left(V_{T} \cup V_{I}\right), \\
\sum_{e \in E_{1}(v)} \sum_{c \in C(e)} \omega_{d e c}^{f}+o u t_{d} \cdot \sum_{e \in E_{3}(v) c \in C(e)} \sum_{d e c}^{f} \leq 0 \\
\forall d \in D, f \in F, v \in \overline{S D(d)} \cap\left(V_{M} \cup V_{V}\right), \\
\sum_{e^{\prime} \in E_{1}(v)} \omega_{e \neq e^{\prime}} \omega_{d e^{\prime} \in C\left(e^{\prime}\right)}^{f}+o u t_{d} \cdot \sum_{e^{\prime \prime} \in E_{3}(v)} \sum_{e \neq e^{\prime \prime} \in C\left(e^{\prime \prime}\right)} \omega_{d e^{\prime \prime} c^{\prime \prime}}^{f} \geq \sum_{c \in C(e)} \omega_{d e c}^{f} \\
\forall d \in D, f \in F, v \in \overline{S D(d)} \cap\left(V_{T} \cup V_{I}\right), e \in E_{1} \cup E_{3}, \\
\sum_{d \in D} \omega_{d e c}^{f} \leq M \cdot \sum_{k \in K(e)} w f a i l^{f k} \cdot \varphi_{e c}^{f k} \quad \forall f \in F, e \in E, c \in C(e), \\
\sum_{k \in K(e)} \varphi_{e c}^{f k} \leq 1 \quad \forall f \in F, e \in E, c \in C(e),
\end{gathered}
$$

$$
\begin{aligned}
& \sum_{e \in E} \sum_{c \in C(e)} \sum_{k \in K(e)} p a t h_{l}^{k} \cdot \varphi_{e c}^{f k} \leq w_{l} \quad \forall f \in F, l \in L_{O}, \\
& \sum_{d \in D} \omega_{d e c}^{f} \leq M \cdot \sum_{s \in S(v)} m f a i l l^{f v s} \cdot \psi_{e c}^{f v s} \\
& \forall f \in F, e \in E, c \in C(e), v \in I(e), \\
& \sum_{s \in S(v)} \psi_{e c}^{f v s} \leq 1 \quad \forall f \in F, e \in E, c \in C(e), v \in I(e), \\
& \sum_{e \in E(v)} \sum_{c \in C(e)} \psi_{e c}^{f v s} \leq 1 \quad \forall f \in F, v \in V, s \in S, \\
& \sum_{d \in D} b_{d} \cdot \omega_{d e c}^{f}-M \cdot\left(1-\psi_{e c}^{f v s}\right) \leq \tau^{f v s} \\
& \forall v \in V \backslash V_{V}, s \in S(v), e \in E(v), c \in C(e), f \in F, \\
& { }_{\tau}^{f v s} \leq \sum_{i \in P T} p k_{i} \cdot \rho_{i}^{v s} \quad \forall v \in V \backslash V_{V}, s \in S(v), f \in F, \\
& \sum_{i \in P T} \rho_{i}^{v s} \leq 1 \quad \forall v \in V \backslash V_{V}, s \in S(v), \\
& \sum_{s \in S(v)} \tau^{f v s} \leq \sum_{j \in R T} r k_{j} \cdot \pi_{j}^{v} \quad \forall v \in V \backslash V_{V}, f \in F, \\
& \sum_{s \in S(v)} \sum_{i \in P T} \rho_{i}^{v s} \leq \sum_{j \in R T} r p k_{j} \cdot \pi_{j}^{v} \quad \forall v \in V \backslash V_{V}, f \in F, \\
& \sum_{j \in R T} \pi_{j}^{v} \leq 1 \quad \forall v \in V \backslash V_{V}, \\
& \sum_{v \in I(e)} \sum_{s \in S(v)}\left(1-m f a i l^{f v s}\right) \cdot \psi_{e c}^{0 v s}+M \cdot\left(1-\omega_{d e c}^{f}\right) \geq \gamma_{d}^{f} \\
& \forall d \in D, f \in F-\{0\}, e \in E, c \in C(e), \\
& \sum_{c \in C(e)} \omega_{d e c}^{0}-\sum_{c \in C(e)} \omega_{d e c}^{f} \leq\left(1-\gamma_{d}^{f}\right) \quad \forall d \in D, f \in F, e \in E, \\
& \sum_{c \in C(e)} \omega_{d e c}^{0}-\sum_{c \in C(e)} \omega_{d e c}^{f} \geq\left(\gamma_{d}^{f}-1\right) \quad \forall d \in D, f \in F, e \in E, \\
& \gamma_{d}^{f}, \omega_{d e c}^{f}, \varphi_{e c}^{f k}, \psi_{e c}^{f v s}, \rho_{i}^{v s}, \pi_{j}^{v} \in\{0,1\}, \tau^{f v s} \in \mathrm{Z}^{+}
\end{aligned}
$$

The objective function (3) minimizes the network CAPEX. Constraints (4)-(8) deal with routing and aggregating demands over the virtual topology. Constraints (4) and (5) ensure that demands not belonging to and belonging to $V_{V}$, respectively, are routed under any failure scenario. In these constraints, demands in $D_{2}$ can use optical by-passes, directly connecting metro-to-metro or metro-to-interconnection nodes. Constraint (6) avoids cycles and ensures that transit and interconnection nodes are used as intermediate nodes in the route of the demands. In contrast, constraint (7) prevents the use of metro and virtual nodes for routing demands. Finally, constraint (8) guarantees that a route over the virtual topology is computed for each demand.

Constraints (9)-(14) connect virtual and optical topologies together. More specifically, constraint (9) ensures that each channel transporting traffic in a virtual link is assigned to a WSON route. Under a specific failure scenario, every used channel must be associated to a failure-free optical route. The number of routes assigned to a channel is limited to 1 , as defined in constraint (10). Additionally, constraint (11) makes sure that the WSON capacity is not exceeded. Constraint (12) guarantees that both ends of each used channel are assigned to some ports in the adjacent nodes. Constraint (13) makes sure 
that, at most, 1 slot is assigned to each end of a channel. In a complementary way, constraint (14) ensures that a slot is assigned to, at most, one channel.

Constraints (15)-(20) dimension the IP/MPLS network taking as inputs all possible assignments produced by constraints (9)-(14). The maximum amount of traffic routed through a slot is computed in constraint (15), whilst constraint (16) provides the slot with enough bit-rate and constraint (17) ensures that only one port is equipped per slot. The required switching capacity of each node is computed in constraint (18), whereas the required capacity in number of ports is obtained by constraint (19). Then, the node class to be equipped in a node must ensure both switching capacity and number of port constraints. Additionally, constraint (20) guarantees that only one router is installed in a node.

When a failure occurs, the non-affected IP/MPLS demands must remain in their current virtual route. In contrast, WSON route and/or OE port assignment changes are allowed. Constraint (21) fixes which demands must remain in their route under every failure scenario, and constraints (22) and (23) prevent these IP/MPLS routes from changing.

Finally, Eq. (24) defines the variables as binary or positive integers.

\section{ILP-Based Algorithm for the Overlay Approach}

The overlay approach is solved by means of two complementary ILPs: one for the IP/MPLS network and another for the WSON. First, the IP/MPLS network is planned by adding redundancy to $\mathrm{OE}$ ports and to transit and interconnection IP/MPLS nodes. One outcome of this optimization is the set of lightpaths to be established in the WSON. Second, for each optical demand, the second ILP finds a pair of link-disjoint routes over the WSON. In the case of a metro-to-transit optical demand, each route connects a metro node to one of the duplicated transit nodes. In the case of transit-to-transit and transit-to-interconnection, each route connects two nodes of the same duplicated transit sub-network, as depicted in Fig. 1.

In addition to the notation defined above, the following sets and parameters are defined:

$P \quad$ Set of optical demands, index $p$.

$K(p) \quad$ Set of WSON routes to support optical demand $p$.

dist $_{e}$ Distance in $\mathrm{km}$ of the shortest WSON route supporting virtual link $e$.

$C_{\text {unp }} \quad$ Cost per kilometer of unprotected lightpath.

Moreover, the set of variables has been extended with

$\delta_{e c i} \quad$ Binary. Equal to 1 if a port of bit-rate $i$ is installed in the end nodes of channel $c$ of virtual link $e$.

$\kappa_{p}^{k q} \quad$ Binary. Equal to 1 if lightpath $q$ of optical demand $p$ belongs to WSON route $k, 0$ otherwise. Two

lightpaths per demand are needed (indices 0 and 1).
Furthermore, Eqs. (1) and (2) are redefined to consider equipment duplication and unprotected lightpath setup:

$$
\begin{gathered}
\mathrm{COST}_{\text {Equipment }}^{\text {Overlay }}=4 \cdot \sum_{e \in E \backslash E_{5}} \sum_{c \in C(e)} \sum_{i \in P T}\left(m p c_{i}+o p c_{i}\right) \cdot \delta_{e c i} \\
+\sum_{v \in V_{M}} \sum_{j \in R T} r c_{j} \cdot \pi_{j}^{v}+2 \cdot \sum_{v \in V_{T} \cup V_{I}} \sum_{j \in R T} r c_{j} \cdot \pi_{j}^{v} \\
\operatorname{COST}_{\text {Lightpaths }}^{\text {Overlay }}=C_{u n p} \cdot \sum_{p \in P} \sum_{k \in K}\left(\kappa_{p}^{k 0}+\kappa_{p}^{k 1}\right) \\
\times \sum_{l \in L} \text { len }_{l} \cdot p a t h_{l}^{k} .
\end{gathered}
$$

Then, we propose the following ILP formulation for the IP/MPLS network planning sub-problem:

\section{Overlay IP/MPLS}

$$
\begin{gathered}
\text { Minimize } \operatorname{COST}_{\text {Equipment }}^{\text {Overlay }}+C_{\text {unp }} \\
\times \sum_{e \in E \backslash E_{5}} \sum_{c \in C(e)} \sum_{i \in P T} d_{i s t_{e}} \cdot \delta_{e c i}
\end{gathered}
$$

subject to:

constraints (4) to (8), where $F=\varnothing$, and constraint (20),

$$
\begin{aligned}
& \sum_{d \in D} B_{d} \cdot \omega_{d e c} \leq \sum_{i \in P T} p k_{i} \cdot \delta_{e c i} \quad \forall e \in E \backslash E_{5}, c \in C(e), \\
& \sum_{i \in P T} \delta_{e c i} \leq 1 \quad \forall e \in E \backslash E_{5}, c \in C(e), \\
& \sum_{e \in E(v) \backslash E_{5}} \sum_{d \in D} B_{d} \cdot \omega_{d e} \leq \sum_{j \in R T} r k_{j} \cdot \pi_{j}^{v} \quad \forall v \in V \backslash V_{V}, \\
& 2 \cdot \sum_{e \in E(v) \backslash E_{5}} \sum_{c \in C(e)} \delta_{e c i} \leq \sum_{j \in R T} r p k_{j} \cdot \pi_{j}^{v} \quad \forall v \in V_{M}, \\
& \sum_{e \in E(v) \backslash E_{5}} \sum_{c \in C(e)} \delta_{e c i} \leq \sum_{j \in R T} r p k_{j} \cdot \pi_{j}^{v} \quad \forall v \in V_{T} \cup V_{I}, \\
& \delta_{e c i} \in\{0,1\} .
\end{aligned}
$$

The cost function in (27) minimizes the cost of the IP/MPLS nodes and OE ports plus an approximation of the cost of the underlying lightpaths based on the shortest WSON routes for each used virtual link. As a consequence of OE port and node duplication, no failure scenarios are considered in the overlay model. Then, constraints (4)-(8) are applied to the non-failure scenario for routing and aggregating demands. Note that the variable $\omega$ used for routing here becomes $\omega_{d e c}$ instead of $\omega_{d e c}^{f}$. Constraint (28) deals with the dimensioning of $\mathrm{OE}$ ports. In contrast to the model proposed for the joint approach, here OE port dimensioning is performed in the virtual links without assigning channels to slots, thereby considerably reducing the problem size. Moreover, constraint (29) ensures that each used channel is equipped with only one couple of ports. Constraints (30)-(32) together with constraint (20) are responsible for dimensioning the nodes. Constraint (30) gets the minimum switching capacity for metro, transit, and interconnection nodes. The minimum number of ports is computed in constraints (31) and (32) for metro and for transit and interconnection nodes, respectively. Note that ports are duplicated in metro nodes. Finally, Eq. (33) defines the new variable as binary. 
The optical demands routing sub-problem can be formulated as follows:

\section{Overlay WSON}

$$
\text { Minimize } \quad \mathrm{COST}_{\text {Lightpaths }}^{\text {Overlay }}
$$

subject to:

$$
\begin{gathered}
\sum_{k \in K(p)} \kappa_{p}^{k q}=1 \quad \forall p \in P, q \in\{0,1\}, \\
p a t h_{l}^{k} \cdot\left(\kappa_{p}^{k 0}+\kappa_{p}^{k 1}\right) \leq 1 \quad \forall p \in P, k \in K(p), l \in L_{o}, \\
\sum_{p \in P} \sum_{k \in K(p)} p a t h_{l}^{k} \cdot\left(\kappa_{p}^{k 0}+\kappa_{p}^{k 1}\right) \leq w_{l} \quad \forall l \in L_{o}, \\
\kappa_{p}^{k q} \in\{0,1\} .
\end{gathered}
$$

The objective function (34) minimizes the total unprotected lightpath cost. Constraint (35) ensures that a couple of lightpaths is found for each optical demand, whereas constraint (36) makes sure that the lightpaths are link disjoint. Constraint (37) guarantees that the capacity of each link is not exceeded. Finally, Eq. (38) defines the new variable as binary.

Table I shows the algorithm used to solve the SIMULTANEO problem with the overlay approach.

\section{Complexity Analysis}

The SIMULTANEO problem can be considered NP-hard since simpler multilayer network planning problems without survivability have been proved to be NP-hard (e.g., [8]). Regarding its size, Table II contains expressions to estimate the number of variables and constraints for the models presented above. Additionally, it provides numerical values for the networks presented in Section V.

Although the overlay algorithm can be solved for small instances, the ILP for the joint approach is impractical even in very small networks as a consequence of the large number of failure scenarios to be considered. Moreover, the large size of both ILPs prevents the application of commercial solvers, such as CPLEX [15], to real backbone multilayer networks similar to those described in Section V. Aiming at providing near-optimal solutions with an acceptable computational effort, in the next section heuristic methods to solve the SIMULTANEO problem are presented.

\section{Heuristic Algorithms}

Meta-heuristics coordinate simple heuristics to find goodquality feasible solutions to optimization problems. Among meta-heuristics, BRKGA, a class of GA, has been recently proposed to effectively solve optimization problems, in particular, network related problems such as routing in IP networks and RWA in optical networks [9,16-18]. Compared with other meta-heuristics, BRKGA has provided better solutions in shorter running times. As in GAs, each individual solution is represented by an array of $n$ genes (chromosome), and
TABLE I

OVERLAY APPROACH Algorithm

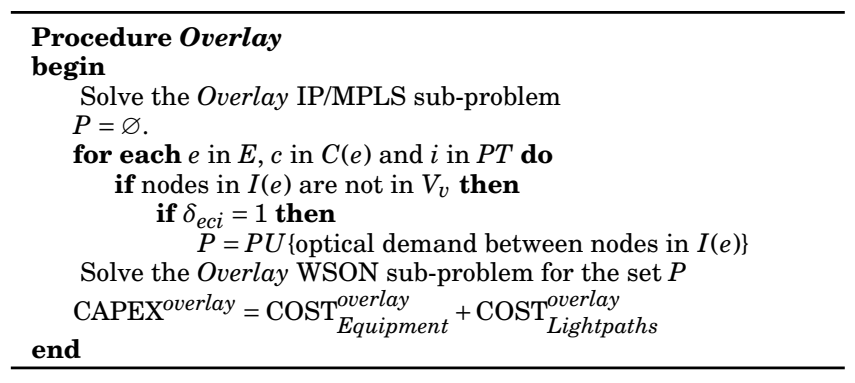

TABLE II

Sizes of THE Models For Both ApproacheS

\begin{tabular}{lll}
\hline Approach & Variables & Constraints \\
\hline Joint & $|F| \cdot|E| \cdot|C| \cdot(|D|+|K|)$ & $|F| \cdot|E| \cdot|D| \cdot|C|$ \\
& $\left(10^{10}\right)$ & $\left(10^{9}\right)$ \\
Overlay & $|D| \cdot|E|+|K| \cdot|P|$ & $|D| \cdot|V| \cdot|E|+|P| \cdot|K| \cdot|L|$ \\
& $\left(10^{4}\right)$ & $\left(10^{5}\right)$ \\
\hline
\end{tabular}

each gene can take any value in the real interval [0, 1]. Each chromosome encodes a solution of the problem and a fitness value, i.e., the value of the objective function. A set of $p$ individuals, called a population, evolves over a number of generations. At each generation, individuals of the current generation are selected to mate and produce offspring, making up the next generation. In BRKGA, individuals of the population are classified into two sets: the elite set $p_{e}$, those individuals with the best fitness values, and the non-elite set. Elite individuals are copied unchanged from one generation to the next, thus keeping track of good solutions. The majority of new individuals are generated by crossover combining two elements, one elite and another non-elite, selected at random. An inheritance probability $\left(\rho_{e}\right)$ is defined as the probability that an offspring inherits the gene of its elite parent. Finally, to escape from local optima a small number of mutant individuals (randomly generated) to complete a population are introduced at each generation. A deterministic algorithm, named decoder, transforms any input chromosome into a feasible solution of the optimization problem and computes its fitness value.

In the BRKGA framework, the only problem-dependent parts are the chromosome internal structure and the decoder, and, thus, one only needs to define them to completely specify a BRKGA heuristic. Since the SIMULTANEO problem primarily consists in routing a set of demands over a virtual topology, we need one gene for each virtual link and for each IP/MPLS node. These genes are used to compute the metric of each virtual link and each IP/MPLS node which is later used for routing demands, i.e., shortest paths are computed with respect to these metrics. Besides, since the order in which the demands are routed influences the goodness of the solution, additional genes are needed to specify the order in which the demands are routed. In this regard, we use one additional gene for each demand which is used to sort the set of demands. Therefore, given a virtual network represented by the graph $G(V, E)$, each individual is represented by an array of $|V|+|E|+|D|$ genes.

To decode chromosomes into feasible solutions, we have designed the algorithm presented in Table III for the joint 
TABLE III

DeCODER Algorithm FOR THE JOINT APPROACH

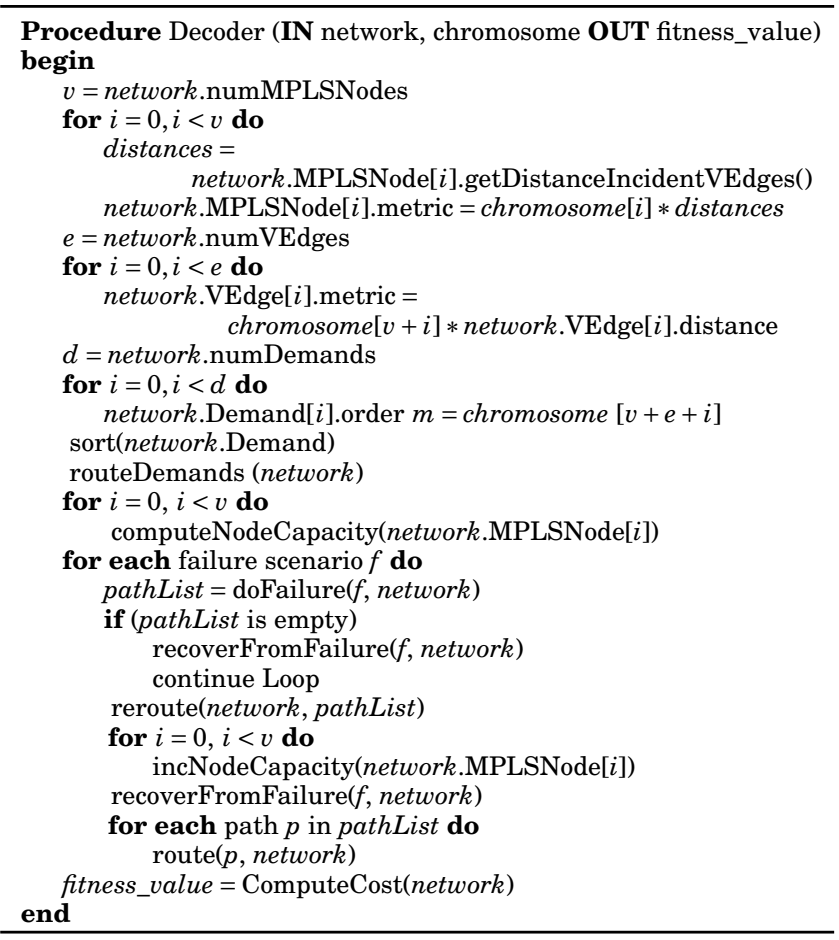

approach. As shown, metrics of IP/MPLS nodes and virtual links are initialized using the assigned gene of the input chromosome, whereas the order in which each demand will be routed is given by the remaining genes. After initializing the elements, the network is dimensioned through routing the set of demands. A solution for the non-failure scenario is obtained at this step. From this basic solution, the set of single-failure scenarios is built. For each failure scenario, we remove the element in failure from the network and compute the list of affected IP/MPLS paths, each path being subsequently rerouted. If additional $\mathrm{OE}$ ports need to be installed in the IP/MPLS nodes, the feasibility of the solution is checked to ensure that the capacity of the node, in terms of number of ports, is not exceeded.

It is worth mentioning that in the case of fiber link or OE port failure, lightpath restoration is tried as a first option during the list of affected paths computation phase. If a lightpath can be restored using the pre-defined set of restoration routes, the associated virtual link and, thus, every MPLS LSP using it, is automatically restored. On the contrary, MPLS LSPs are rerouted over the resulting virtual topology, hence possibly increasing both IP/MPLS nodes' switching capacity and OE ports.

Demand routing is mainly performed over a virtual topology which is pre-computed beforehand over the given network topology. Virtual links are created between pairs of IP/MPLS nodes (connecting metro-to-transit, transit-to-transit, and transit-to-interconnection nodes) that satisfy their distance being lower than a given threshold. For each virtual link, a set of routes over the optical network is computed: the shortest one and a number of restoration routes, one route for each optical link in the shortest route. Then, a failure in an optical link
TABLE IV

BRKGA PARAMETER VALUES

\begin{tabular}{lll}
\hline$n$ & length of the chromosome & $|V|+|E|+|D|$ \\
$p$ & size of population & $\min \{50, n\}$ \\
$p_{e}$ & size of elite population & $0.2 * p$ \\
$p_{m}$ & size of mutant population & $0.2 * p$ \\
$\rho_{e}$ & elite inheritance probability & 0.7 \\
\hline
\end{tabular}

affecting a virtual link can be recovered at the optical layer by restoring the underlying lightpath.

A set of feasible routes is then pre-computed for each demand. Here, we use a $k$-shortest path algorithm to pre-compute distinct routes. Two subsets of routes are pre-computed, one over the virtual topology and another over the optical topology, thus enabling optical by-passing. During the decoder process, route metric re-computation is performed, ensuring that the shortest route, in terms of the metric, is chosen at each step.

We performed several tests to tune the parameters used in the algorithm. Table IV shows the final values. It is worth pointing out that, as a consequence of the size of the problems, the length of the chromosome was higher than 400 genes. By choosing the size of the population equal to the length of the chromosome, the decoder algorithm took more than $50 \mathrm{~ms}$ to decode the solution from any chromosome and, consequently, more than $15 \mathrm{~s}$ to build one generation. This greatly worsened the convergence properties of the algorithm. Hence, we reduced the size of the population so as to bring convergence within acceptable values.

The heuristic for the overlay approach uses a similar algorithm for the decoder, where the overlay network is obtained from the non-failure scenario and applying IP/MPLS node and OE port redundancy, as explained above. Moreover, pairs of link-disjoint optical routes need to be pre-computed during the virtual topology definition.

The performance of the proposed heuristics was compared against the optimal solution obtained solving the mathematical models described in Section III, over small multilayer topologies. In all the tests performed, the optimal solution was found within running times of some seconds, in contrast to several hours needed to find the optimal solution with the models.

\section{ILLUSTRATIVE NUmERICAL RESUlts}

The performance of the joint approach has been compared to that of the overlay approach in terms of CAPEX. To this end, both approaches have been applied over three national optical network topologies with different IP/MPLS topologies on the top. More precisely, we have considered three optical network topologies: the 21-node Spanish Telefónica (TEL) topology, the 20-node British Telecom (BT) topology, and the 21-node Deutsche Telecom (DT) topology. Figure 3 shows the topologies under consideration.

Aiming at applying the heuristic algorithms over a wide range of multilayer networks, on top of the optical topologies, different IP/MPLS topologies with 40 metro nodes and different numbers of transit and interconnection nodes are 

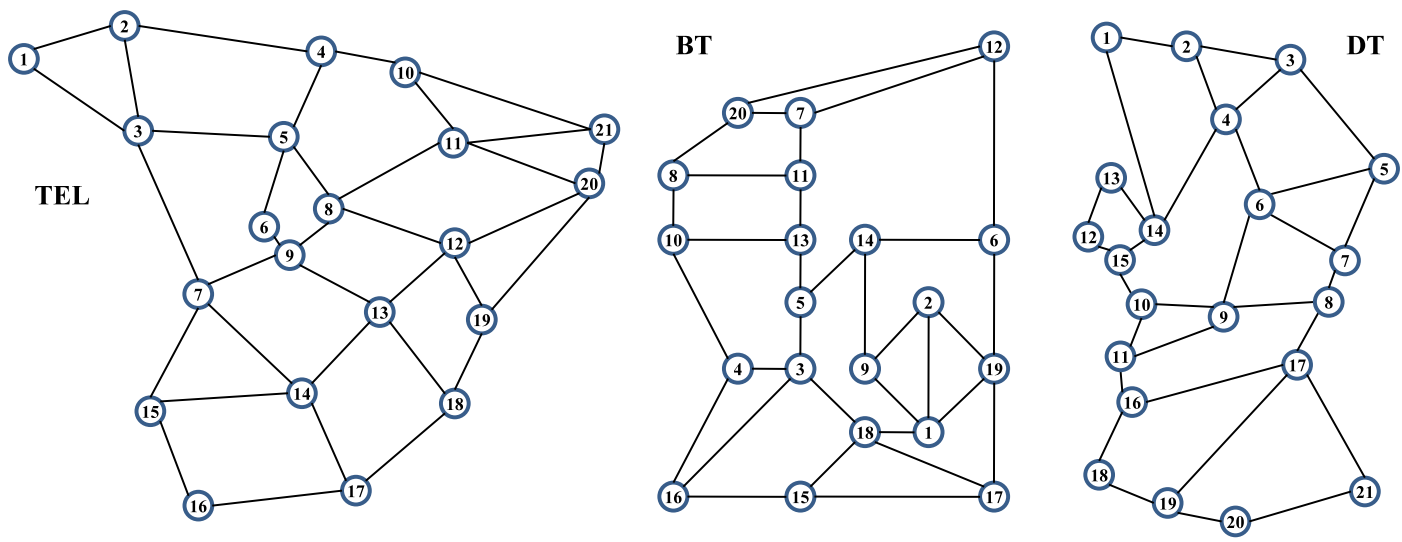

\begin{tabular}{|l|c|c|c|c|}
\hline Net & Transit & Interconn. & $\begin{array}{c}\text { Metro coverage } \\
\text { degree }\end{array}$ & $\begin{array}{c}\text { Traffic mix } \\
\text { (National/Interconnection) }\end{array}$ \\
\hline TEL & $3,4,9,11,14,15,19,21$ & $6,8,20$ & $0.1 \%$ & $30 \% / 70 \%$ \\
\hline BT & $1,9,10,12,14,16,20$ & $7,13,15,19$ & $30 \%$ & $40 \% / 60 \%$ \\
\hline DT & $3,4,5,8,9,14,19,21$ & $6,7,10,13,20$ & $90 \%$ & $50 \% / 50 \%$ \\
\hline
\end{tabular}

Fig. 3. (Color online) Sample optical network topologies used in this paper: 21-node Spanish Telefónica (left), 20-node British Telecom (center), and 21-node Deutsche Telecom (right). A table with details of the IP/MPLS topologies as well as the IP/MPLS traffic mix is also provided.

TABLE V

Cost of IP/MPLS Nodes AND OE Ports (C.U.)

\begin{tabular}{llllll}
\hline & Class 1 & Class 2 & $\begin{array}{l}\text { Class } 3 \\
640\end{array}$ & $\begin{array}{l}\text { Class } 4 \\
1280\end{array}$ & $\begin{array}{l}\text { Class } 5 \\
2560\end{array}$ \\
$\begin{array}{l}\text { Capacity } \\
\text { (Gbps) }\end{array}$ & 160 & 320 & & & \\
$\begin{array}{l}\text { Max. ports } \\
\text { Cost }\end{array}$ & 4 & 8 & 16 & 32 & 64 \\
\hline & 3 & 4.5 & 6.5 & 22.5 & 50.19 \\
\hline & & 1 Gbps & $10 \mathrm{Gbps}$ & $40 \mathrm{Gbps}$ & $\begin{array}{l}100 \mathrm{Gbps} \\
\text { Port in IP/MPLS }\end{array}$ \\
$\begin{array}{l}\text { node } \\
\text { Port in OXC }\end{array}$ & 0.35 & 1.25 & 7.625 & 20.625 \\
\hline
\end{tabular}

designed. The table in Fig. 3 specifies the locations of transit and interconnection nodes (identified by the associated OXCs) of each multilayer network. Moreover, the spatial positions of metro nodes are characterized by a uniform coverage degree based on the Kolmogorov-Smirnov goodness-of-fit test [19]. A value close to $100 \%$ indicates metro nodes uniformly located around every OXC node, whereas a low value denotes the presence of areas with high densities of metro nodes. Figure 3 also contains the coverage degree of the three networks under study. Regarding traffic, we assume two types of demands: national, where both metro end nodes belong to the network, and interconnection, where one of the end nodes is outside the network. The considered traffic mix is also detailed in Fig. 3. As shown, three different multilayer network scenarios can be identified, from an unbalanced scenario where $70 \%$ of the total is interconnection traffic with only 3 interconnection nodes and several high density metro areas, to the well-balanced scenario with $50 \%$ of interconnection traffic, 5 interconnection nodes, and near-uniform metro areas.

Each multilayer network has been planned for six gradually increasing traffic loads, starting from an initial load of 4 Gbps per metro node and with increments of $45 \%$ at each step (roughly representing a year-over-year traffic increase). Aiming at providing accuracy, each traffic load has been executed 10 times with randomly generated demands following the above characteristics.

To compute the network CAPEX, we consider an adaptation of the equipment costs proposed in [20]. Table V provides the costs used in cost units (c.u.) for IP/MPLS nodes and OE ports. However, to the best of our knowledge, no study provides the cost of using an already deployed WSON infrastructure. In view of this, Fig. 4 plots the CAPEX savings obtained by implementing the joint network approach for various costs of unprotected lightpaths $C_{u n p}$ ranging from 0 to 0.5 c.u. Each point in Fig. 4 has been obtained after computing the solution for both approaches on a set of 10 randomly generated executions for each of the considered loads.

As shown, the joint approach provides CAPEX savings even when the costs of using the WSON infrastructure are not considered (i.e., $C_{\text {unp }}=0$ ), meaning that the cost of the IP/MPLS layer is cheaper with the joint network approach. However, and for the sake of a fair comparison, we consider $C_{\text {unp }}=0.1$, which provides similar prices for WSON and for $\mathrm{OE}$ ports when computing lightpath costs. Regarding the cost of restorable lightpaths $C_{\text {rest }}$, values ranging from 1.33 to 2 with respect to the cost of unprotected lightpaths are considered in Fig. 4. As depicted, the unbiased $C_{\text {rest }} / C_{\text {unp }}$ ratio of 1.5 , in between the unprotected and the $1+1$ protected costs, provides CAPEX savings ranging from $13 \%$ to $24 \%$ when the joint network approach is implemented.

Aiming at performing an in-depth analysis of the network structure provided by both approaches, Fig. 5 shows a set of graphs with the switching capacity of the IP/MPLS nodes and the installed $\mathrm{OE}$ ports as a function of the network load. Similar behavior can be observed in all networks under consideration and for each approach. Regarding switching capacity (Fig. 5 left), both approaches equip IP/MPLS metro nodes with the same capacity since it only depends on the incoming traffic. On the contrary, the capacity of transit and 

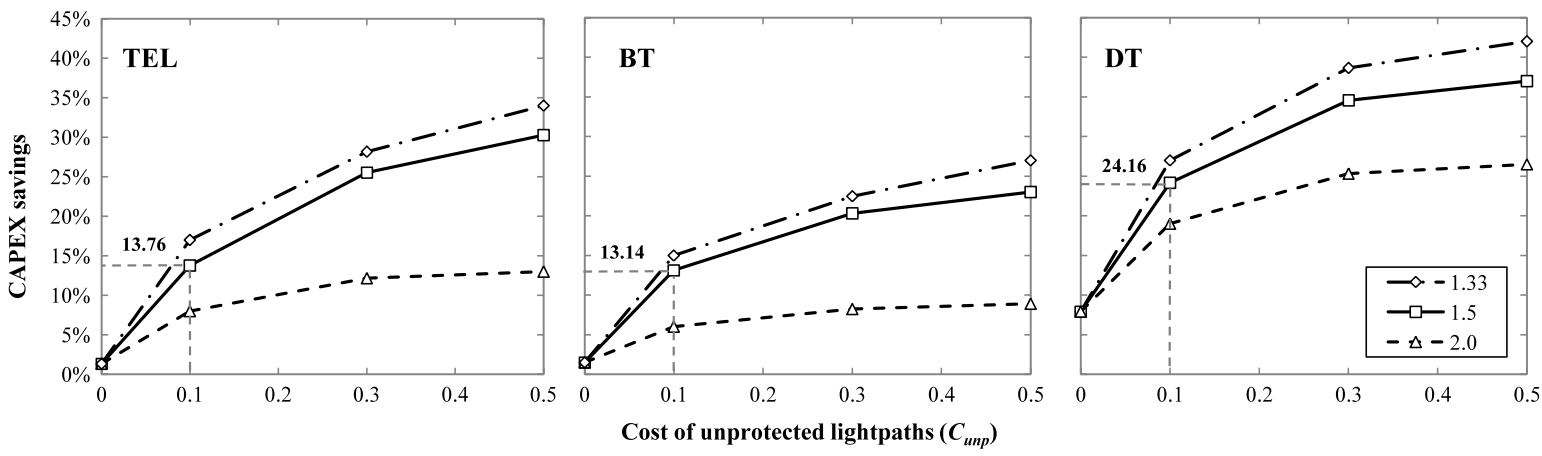

Fig. 4. Average CAPEX savings for several $C_{r e s t} / C_{u n p}$ ratios by implementing the joint network approach as a function of the cost per $\mathrm{km}$ of unprotected lightpaths in the TEL (left), BT (center), and DT (right) networks.
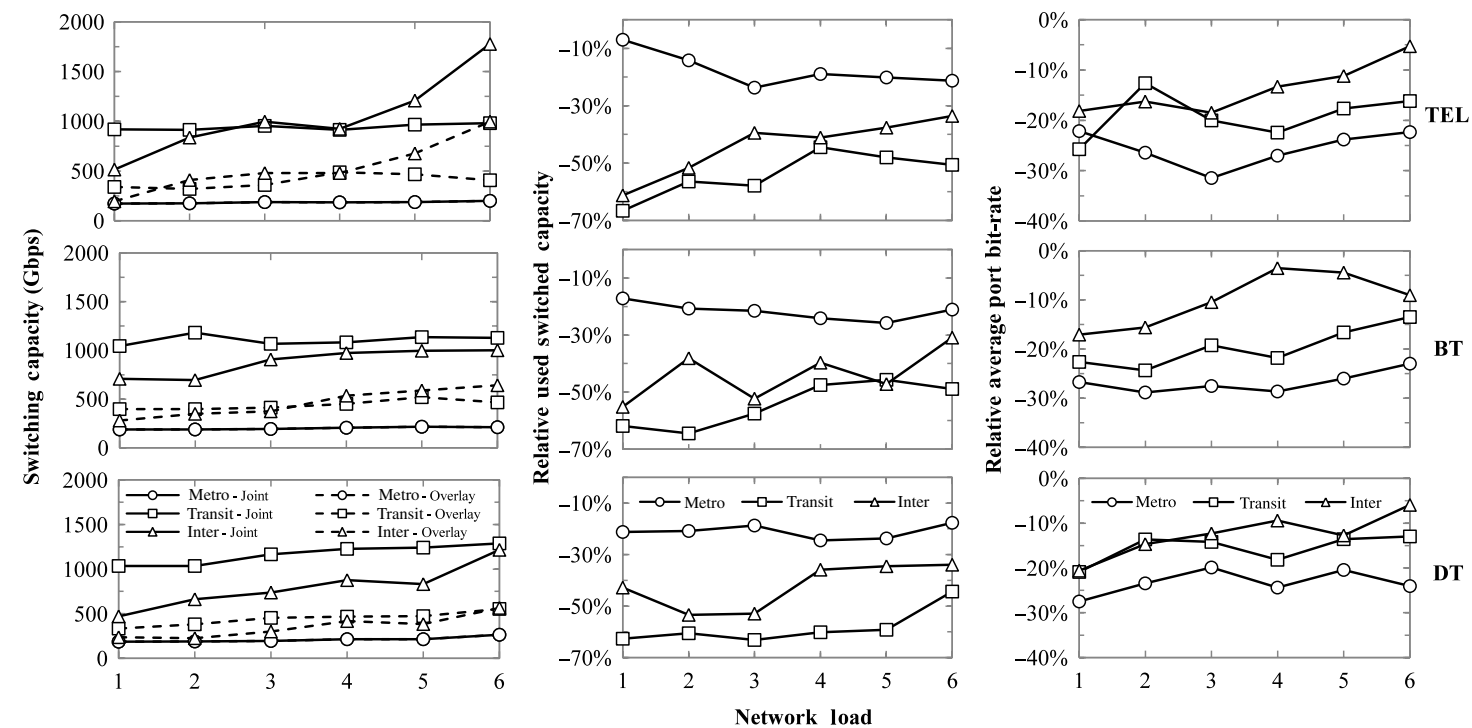

Fig. 5. Switching capacity of IP/MPLS nodes (left), relative joint-overlay used switching capacity (center), and relative joint-overlay average port bit-rate (right) as a function of the network load in the TEL (top), BT (middle), and DT (bottom) networks. Each load (4 Gbps $* 1.45^{(i-1)}$ ) is identified by the exponent $i$.

interconnection nodes is higher in the joint approach as a result of both the extra capacity and the OE ports added to deal with failures. To illustrate this, Table VI shows the number and average bit-rate of OE ports installed under the non-failure and failure scenarios, for the networks obtained under both approaches. It is worth noting that, while both the number and the average bit-rate of OE ports is the same under the non-failure scenario, the joint approach needs more OE ports for recovery purposes. However, due to the recovery mechanism proposed (lightpath restoration first, connectivity recovery second, and LSP reroute third), the average bit-rate used for recovery in the joint approach is lower than in the overlay case, which simply duplicates OE ports. Note that when lightpaths can be recovered at the optical layer, virtual links are automatically restored, avoiding node over-dimensioning or the addition of extra OE ports. In the case where only a subset of lightpaths can be restored after a failure, those using higher bit-rate (and more expensive) OE ports are restored first. The remaining lower rate lightpaths are then removed and connectivity recovery is performed, adding then extra capacity and OE ports.

Supporting the previous statement, Fig. 5 (center) shows the switched capacity used under the non-failure scenario in relative values. Nodes in the joint approach have higher unused switching capacity than those in the overlay approach. Also, as a consequence of the recovery mechanism proposed, the joint approach uses OE ports with lower average bit-rate than the overlay approach (Table VI and Fig. 5 right).

Finally, Fig. 6 shows the evolution, in terms of relative gap to the best solution of the heuristic algorithm found in a $10 \mathrm{~h}$ run, considering an intensity $i=4$, and for the three networks under consideration. In this figure, one can observe the amount of time that the BRKGA requires to reach convergence and thus the complexity of the problem for the joint approach. In this regard, a brief analysis of the proposed network instances identifies differences in the complexities of the problems. For instance, the sizes of the virtual topologies are 326, 361, and 408 virtual links for the TEL, BT, and DT networks 
TABLE VI

OE Ports ANALYSIS (TEL NETWORK; INTENSITY $i=4$ )

\begin{tabular}{llllll}
\hline & \multicolumn{2}{c}{ Joint } & & \multicolumn{2}{c}{ Overlay } \\
\cline { 2 - 3 } \cline { 5 - 6 } & Num. & $\begin{array}{l}\text { Average bit- } \\
\text { rate (Gbps) }\end{array}$ & & Num. & $\begin{array}{l}\text { Average bit- } \\
\text { rate (Gbps) }\end{array}$ \\
\hline Non-failure & 134 & 67.94 & & 134 & 67.94 \\
Recovery & 194 & 42.41 & & 134 & 67.94 \\
TOTAL & 328 & 52.84 & 268 & 67.94 \\
\hline
\end{tabular}

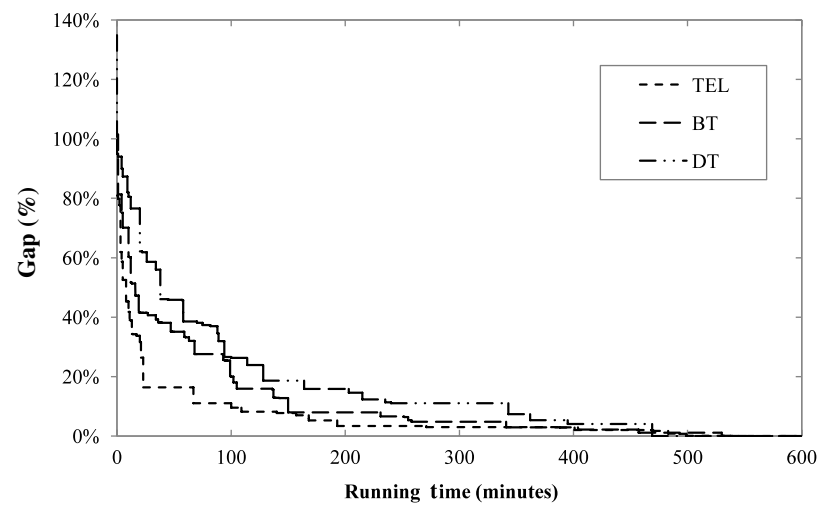

Fig. 6. Gap to the best solution against heuristics running time.

respectively. Thus, the mean number of feasible routes for a given demand significantly increases from the TEL to the DT network, which, as shown, affects the convergence time.

\section{CONCLUSIONS}

This work addressed the design of survivable IP/MPLSover-WSON multilayer networks against single IP/MPLS node, OE port, or fiber link failures. To this goal, two alternative approaches were compared with the objective of CAPEX minimization: the proposed joint approach consisting of over-dimensioning backbone IP/MPLS nodes and applying lightpath and connectivity recovery, and the conventional overlay approach consisting of duplicating backbone IP/MPLS nodes. ILP models for both approaches were presented and heuristic algorithms to obtain near-optimal solutions were developed in view of the fact that the proposed ILP models become impractical if realistic problem instances are considered.

The performance of both approaches was extensively assessed considering three national optical network topologies with different IP/MPLS topologies on the top. From the results obtained, it can be concluded that the proposed joint approach leads to CAPEX savings as high as $24 \%$ in the DT network and $13 \%$ in the TEL and BT networks, when compared with the overlay approach. Analyzing the structure of the networks obtained under both approaches, the joint approach over-dimensions IP/MPLS nodes, not only to deal with failures, but also as a consequence of the extra OE ports required in each node for recovery purposes. However, the average bit-rate of the extra OE ports in the joint approach is lower than that of those needed in the non-failure scenario as a result of the recovery strategy proposed.

As a final remark, it is worth pointing out that since the overlay approach duplicates both IP/MPLS transit and interconnection nodes and $\mathrm{OE}$ ports, the resulting networks require higher operational costs than those in the joint approach. Moreover, extra OE ports added for recovery purposes in the joint approach can remain switched-off while not used, thus leading to the additional benefit of energy savings.

\section{ACKNOWLEDGMENTS}

The research leading to these results has received funding from the European Community's Seventh Framework Programme FP7/2007-2013 under grant agreement no. 247674 STRONGEST project. Moreover, it was supported by the Spanish Science Ministry through the project ENGINE (TEC2008-02634).

\section{REFERENCES}

[1] A. Chiu and J. Strand, "Joint IP/optical layer restoration after a router failure," in Proc. OFC 2001, Anaheim, CA, USA, Mar. 2001.

[2] P. Chołda and A. Jajszczyk, "Recovery and its quality in multilayer networks," J. Lightwave Technol., vol. 28, pp. 372-389, Feb. 2010.

[3] C. Chigan, G. W. Atkinson, and R. Nagarajan, "Cost effectiveness of joint multilayer protection in packet-over-optical networks," $J$. Lightwave Technol., vol. 21, pp. 2694-2704, Nov. 2003.

[4] L. Velasco, F. Agraz, R. Martínez, R. Casellas, S. Spadaro, R. Muñoz, and G. Junyent, "GMPLS-based multi-domain restoration: Analysis, strategies, policies and experimental assessment," J. Opt. Commun. Netw., vol. 2, pp. 427-441, July 2010.

[5] K. Zhu and B. Mukherjee, "Traffic grooming in an optical WDM mesh network," IEEE J. Sel. Areas Commun., vol. 20, pp. 122-133, 2002.

[6] B. Chen, G. Rouskas, and R. Dutta, "On hierarchical traffic grooming in WDM networks," IEEE/ACM Trans. Netw., vol. 16, pp. 1226-1238, 2008.

[7] B. Chen, G. Rouskas, and R. Dutta, "Clustering methods for hierarchical traffic grooming in large-scale mesh WDM networks," J. Opt. Commun. Netw., vol. 2, pp. 502-514, 2010.

[8] X. Zhang, F. Shen, L. Wang, S. Wang, L. Li, and H. Luo, "Two-layer mesh network optimization based on inter-layer decomposition," Photon. Netw. Commun., vol. 21, pp. 310-320, 2010.

[9] J. Gonçalves and M. Resende, "Biased random-key genetic algorithms for combinatorial optimization," J. Heuristics, posted Aug. 2010 , in press.

[10] R. Morais, C. Pavan, A. Pinto, and C. Requejo, "Genetic algorithm for the topological design of survivable optical transport networks," J. Opt. Commun. Netw., vol. 3, pp. 17-26, Jan. 2011.

[11] I. de Miguel, R. Vallejos, A. Beghelli, and R. Durán, "Genetic algorithm for joint routing and dimensioning of dynamic WDM networks," J. Opt. Commun. Netw., vol. 1, pp. 608-621, Dec. 2009.

[12] E. Mannie, Ed., "Generalized multi-protocol label switching (GMPLS) architecture," IETF RFC-3945, Oct. 2004 [Online]. Available: http://www.ietf.org/rfc/rfc3945.txt. 
[13] W. Colitti, K. Steenhaut, D. Colle, M. Pickavet, J. Lemeire, and A. Nowé, "Integrated routing in GMPLS-based IP/WDM networks," Photonic Network Commun., vol. 21, pp. 238-252, 2010 .

[14] G. Chiruvolu, A. Ge, D. Elie-Dit-Cosaque, M. Ali, and J. Rouyer, "Issues and approaches on extending Ethernet beyond LANs," IEEE Commun. Mag., vol. 42, pp. 80-86, Mar. 2004.

[15] CPLEX [Online]. Available: http://www-01.ibm.com/software/ integration/optimization/cplex-optimizer/.

[16] T. Noronha, M. Resende, and C. Ribeiro, "A biased random-key genetic algorithm for routing and wavelength assignment," $J$. Global Optim., vol. 50, pp. 503-518, 2010.
[17] R. Reis, M. Ritt, L. Buriol, and M. Resende, "A biased random-key genetic algorithm for OSPF and DEFT routing to minimize network congestion," Int. Trans. Oper. Res., vol. 18, pp. 401-423, 2011.

[18] J. Gonçalves and M. Resende, "A parallel multi population genetic algorithm for a constrained two dimensional orthogonal packing problem," J. Comb. Optim., vol. 22, pp. 180-201, 2010.

[19] C. P. Robert and G. Casella, Monte Carlo Statistical Methods, 2nd ed. Springer, New York, NY, USA, 2004.

[20] R. Huelsermann, M. Gunkel, C. Meusburger, and D. Schupke, "Cost modeling and evaluation of capital expenditures in optical multilayer networks," J. Opt. Netw., vol. 7, pp. 814-833, Sept. 2008. 